\title{
Band gap creation using quasiordered structures based on sonic crystals
}

\author{
V. Romero-García and E. Fuster \\ Grupo de Acústica Arquitectónica y del Medio Ambiente, Departamento de Física Aplicada, Universidad \\ Politécnica de Valencia, Cno. de Vera s/n. 46022 Valencia, Spain \\ L. M. García-Raffi and E. A. Sánchez-Pérez \\ Instituto de Matemática Pura y Aplicada, Departamento de Matemática Aplicada, Universidad Politécnica \\ de Valencia, Cno. de Vera s/n. 46022 Valencia, Spain \\ M. Sopena, J. Llinares, and J. V. Sánchez-Pérez ${ }^{a)}$ \\ Grupo de Acústica Arquitectónica y del Medio Ambiente, Departamento de Física Aplicada, Universidad \\ Politécnica de Valencia, Cno. de Vera s/n. 46022 Valencia, Spain
}

(Received 24 November 2005; accepted 24 March 2006; published online 25 April 2006)

\begin{abstract}
It is well known that sonic crystals are periodic structures that present acoustic band gaps attenuation centered at frequencies related with the lattice constant of the structure. We present an approach based on genetic algorithms to create band gaps in a predetermined range of frequencies. The mechanism used by genetic algorithms to achieve this objective is the creation of vacancies in a starting sonic crystal, built with air-embedded rigid scatterers. As a result of the optimization process, we obtain quasiordered structures on which acoustic properties are based in the multiple scattering theory. (C) 2006 American Institute of Physics. [DOI: 10.1063/1.2198012]
\end{abstract}

Sonic crystals (SCs) are periodic structures with a strong modulation of the elastic constants between the scatterers and the surrounding material. There are two main features of such systems: (1) SCs work as refractive devices ${ }^{1,2}$ due to the fact that, in the low frequency region, they behave as an homogeneous medium with an acoustic impedance greater than that of the host material. (2) SCs present band gaps, ${ }^{3}$ i.e., ranges of sound frequencies in which there is no sound propagation inside the crystal. The presence of these band gaps is explained by well-known Bragg's sound reflections inside the crystals ${ }^{4,5}$ that depend on the lattice constant, i.e., on the geometry of the SC.

The technological use of SC improves the acoustic properties mentioned above. There are studies showing that it is possible to optimize sound attenuation obtained by rigid scatterer based SCs, creating a periodic distribution of vacancies in the array. ${ }^{6}$ In recent years the strategy to enhance SC properties has been based on the use of scatterers with acoustical properties added. For example, the use of resonators as local scatterers causes a strong scattering effect around the resonance frequency, producing a sound attenuation peak independent of the periodicity of the crystal. ${ }^{7-9}$ Also, the attenuation obtained by SC has been improved using scatterers made up of porous absorbent-coated rigid cylinders embedded in air. ${ }^{10}$ Convergent lenses have been built using Helmholtz resonators obtaining very good focusing results. ${ }^{11}$ Some authors have obtained good results using rigid cylinders embedded in a porous medium. ${ }^{12}$

However, the use of SCs as outdoor acoustic barriers requires scatterers made up of robust and long-lasting materials (PVC, wood, or aluminum). This requirement cannot be easily met using the scatterers mentioned above. This is the reason why it seems interesting to analyze the possibility of optimizing the attenuation capability of SCs made with rigid scatterers. An example of this would be to create vacancies in the SC in order to obtain attenuation peaks nondependent

\footnotetext{
${ }^{a)}$ Electronic mail: jusanc@ @is.upv.es
}

on lattice spacing as it occurs in local resonator based SCs.

There are several mathematical tools that can be used for optimizing this kind of structures. An example is the genetic algorithms (GA). ${ }^{13}$ GA are based on the natural selection process and are specially indicated for discrete problems. This procedure can work together with the multiple scattering theory (MST), ${ }^{14,15}$ which is a self-consistent method for calculating acoustic pressure including all orders of scattering. Given a starting SC, the GA generates quasiordered structures (QOSs) offspring that are classified in terms of a fitness function based on the pressure values at a specific point. The simulation of the sound scattered by every structure analyzed by GA is performed by a two-dimensional (2D) MST. After a predetermined number of generations, the algorithm stops selecting the best-fitted QOS. This methodology has been used for the design of acoustic lenses based on SCs, obtaining excellent results. ${ }^{16-18}$

In this letter we present the theoretical results obtained using the methodology mentioned above. This approach allows the design of QOSs made with rigid scatterers that attenuate sound in a predetermined band frequency independently of the geometry of the array. The mechanism used by AG to achieve this objective is the creation of vacancies in the array. We also present experimental results that validate the numerical calculations.

Figure 1 shows the starting SC. It has been designed with the following constraints: A large number of scatterers involves long a CPU computational time, and the SC topology should be simple to enable its implementation for recording measurement purposes. In our case we measure the sound attenuation spectrum, usually called the insertion loss, at the $\Gamma X$ direction $\left(0^{\circ}\right)$, which corresponds to a Bragg's frequency of $3090 \mathrm{~Hz}$. The insertion loss is the difference between the sound level recorded at the same point with and without the sample. The experiments have been performed in an anechoic chamber using a directional white noise sound source. A collimated beam is obtained by placing the source at the focus of a parabolic reflector $(80 \mathrm{~cm}$ diam size $)$. We 


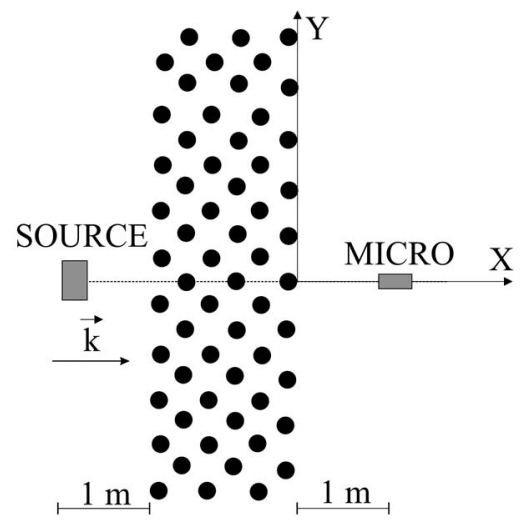

FIG. 1. Starting SCs consisting of hollow cylindrical aluminum rods $1 \mathrm{~m}$ long mounted in a triangular pattern with lattice constant $a=6.35 \mathrm{~cm}$. The diameter of the cylinders is $d=4 \mathrm{~cm}$. The sample under study consists of an array of six rows with ten cylinders each, and a rectangular external shape.

analyze the results with a resolution of $8 \mathrm{~Hz}$.

We are interested in maximizing sound attenuation for both a predetermined sound frequency (objective 1) and a range of sound frequencies (objective 2) at a point of coordinates $(x, y)$ located behind the crystal. In both cases, GA works with mathematical operators (crossover, mutation, and selection) in order to create vacancies at the coordinates of the scatterers $\left(X_{\text {cil }}, Y_{\text {cil }}\right)$. The formula that gives the acoustic attenuation in $\mathrm{dB}$ is

$$
\begin{aligned}
\text { Attenuation }(\mathrm{dB})= & 20 \log \\
& \times\left(\frac{\left|P_{\text {direct }}(1,0)\right|}{\left|P_{\text {interfered }}\left(1,0, X_{\text {cil }}, Y_{\text {cil }}, v, 0.02\right)\right|}\right),
\end{aligned}
$$

where $\left|P_{\text {interfered }}\left(1,0, X_{\text {cil }}, Y_{\text {cil }}, v, 0.02\right)\right|$ is the acoustic pressure with a sample at point $(1,0)$ for frequency $v$ and cylinder radius $=0.02 \mathrm{~m}$. The incident wave has been considered as a plane wave with $\left|P_{\text {direct }}(1,0)\right|=1$. From this formula it is clear that maximizing sound attenuation involves minimizing the interfered pressure. Then the fitness function corresponding to the objective 1 is defined as

$$
\text { Fitness Function }_{1}=\left|P_{\text {interfered }}\left(1,0, X_{\text {cil }}, Y_{\text {cil }}, v, 0.02\right)\right| \text {. }
$$

In order to achieve objective 2 we define the next fitness function as

$$
\text { Fitness Function } 2=\bar{p}+\sum_{j=1}^{N_{v}} \sqrt{\frac{\left(\bar{p}-\left|p_{j}\right|\right)^{2}}{N_{v}^{2}}},
$$

where $N_{v}$ represents the total number of frequencies considered in the range, the term

$$
\bar{p}=\sum_{j=1}^{N_{v}} \frac{\left|p_{j}\right|}{N}
$$

is the mean pressure for these frequencies at point $(1,0)$, and the sum evaluates the mean deviation of the pressure at each frequency with respect to $\bar{p}$.

The GA introduces a stochastic component in the search for the best fitted structure, that is, different runs starting at the same structure generate different optimized QOSs, but in all the cases the value of sound attenuation obtained is similar. Figure 2 shows the results of two different runs corre-

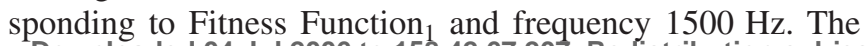

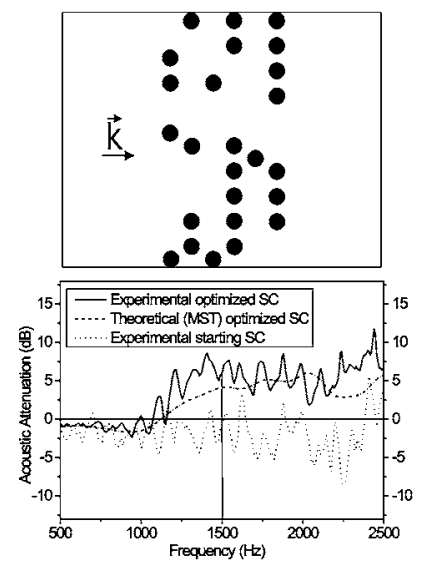

(a)

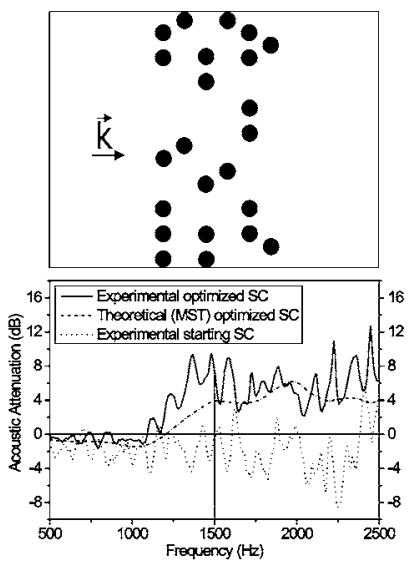

(b)
FIG. 2. Two QOSs optimized using Fitness Function $_{1}$. Here the acoustic attenuation has been maximized to $1500 \mathrm{~Hz}$ at point $(1,0)$. The plots show the experimental insertion loss for the starting SC (continuous line) and the insertion loss obtained in the optimized QOS, experimental (dotted line) and calculated by MST (short dotted line).

optimized QOSs have 41.66\% [Fig. 2(a)] and 38\% [Fig. 2(b)] of the total number of scatterers in the starting SC, but the acoustic attenuation obtained in both cases is about five times higher [6.85 dB in Fig. 2(a) and $6.26 \mathrm{~dB}$ in Fig. 2(b) as opposed to $1.23 \mathrm{~dB}$ in the starting SC]. The quality of the simulations is validated by the good agreement between the theory and the experiments in both cases.

In Fitness Function $_{2}$ we have considered a range of $600 \mathrm{~Hz}$ centered at 1100, 1400, 1700, and $2000 \mathrm{~Hz}$, respectively. Note that in all cases, the considered frequency band lies under Bragg's frequency $(3090 \mathrm{~Hz})$. Figure 3 shows examples of an optimized QOS for each range. Once again the total number of cylinders in the optimized QOSs varies between $40 \%$ and $46 \%$ with respect to the number of cylinders in the starting SC.

Figure 4 shows the attenuation spectra obtained in the case of the QOSs shown in Fig. 3(d). In the optimized QOS an attenuation peak appears in the chosen frequency range that is absent in the starting SC, with the corresponding

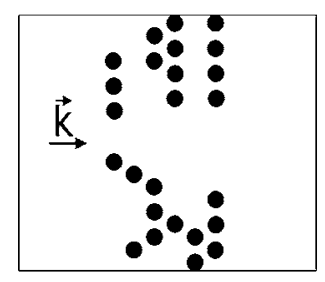

(a)

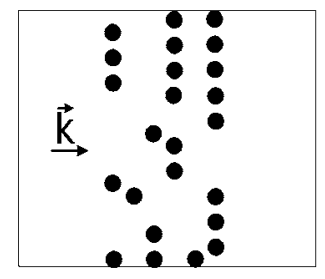

(c)

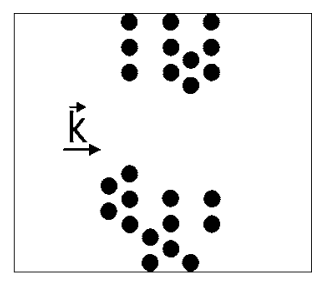

(b)

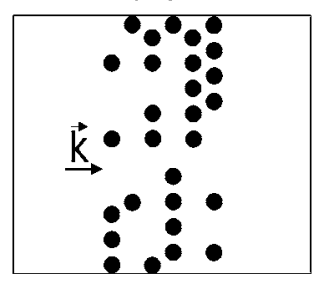

(d)
FIG. 3. QOSs obtained by AG for Fitness Function $_{2}$ in (a) $900-1400 \mathrm{~Hz}$, centroid at $1100 \mathrm{~Hz}$; (b) $1100-1700 \mathrm{~Hz}$, centroid at $1400 \mathrm{~Hz}$; (c) $1400-2000 \mathrm{~Hz}$, centroid at $1700 \mathrm{~Hz}$; and (d) $1700-2300 \mathrm{~Hz}$, centroid at $2000 \mathrm{~Hz}$. 


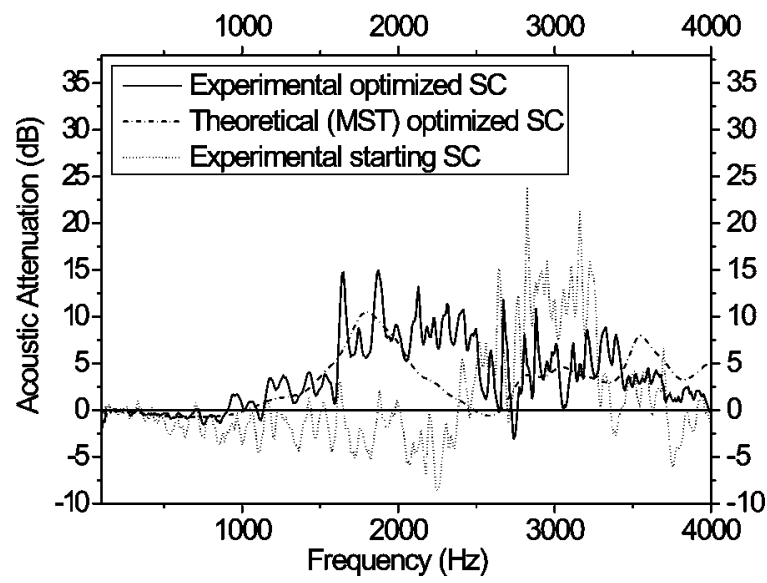

FIG. 4. Attenuation spectra corresponding to Fig. 3(d): experimental insertion loss for the starting SC (dotted line) and the insertion loss obtained for the optimized QOS, experimental (continuous line) and calculated by MST (short-dotted line).

Bragg's peak vanishing at $3090 \mathrm{~Hz}$. This is a feature added with respect to SCs with resonators as scatterers. ${ }^{9}$ Notice that for the starting SC in the frequency range selected, there is only negative attenuation, i.e., sound reinforcement.

In order to compare different QOSs we need a parameter that quantifies the total sound attenuation produced in a range of frequencies. This is the reason why we define the attenuation area (AA) as the area enclosed between the positive attenuation spectra and the $0 \mathrm{~dB}$ threshold in the frequency range selected. Table I shows the AA and the average attenuation values for the four optimized QOSs shown in Fig. 3. Notice that the AA for the starting SC is approximately equal to zero.

TABLE I. AA (attenuation area) and average attenuation for optimized QOSs shown in Figs. 3(a)-3(d).

\begin{tabular}{cccc}
\hline \hline $\begin{array}{c}\text { Range to } \\
\text { optimize } \\
(\mathrm{Hz})\end{array}$ & $\begin{array}{c}\text { Central } \\
\text { frequency } \\
(\mathrm{Hz})\end{array}$ & $\begin{array}{c}\text { Attenuation } \\
\text { area (AA) } \\
(\mathrm{dB} \mathrm{Hz})\end{array}$ & $\begin{array}{c}\text { Average } \\
\text { attenuation } \\
(\mathrm{dB})\end{array}$ \\
\hline$(800-1400)$ & 1100 & 731 & 1.21 \\
$(1100-1700)$ & 1400 & 3411 & 5.61 \\
$(1400-2000)$ & 1700 & 3420 & 5.7 \\
$(1700-2300)$ & 2000 & 5088 & 8.48 \\
\hline \hline
\end{tabular}

We have demonstrated that it is possible to obtain QOSs consisting of rigid scatterers embedded in air that generate large attenuation peaks in ranges of frequency independent of the lattice spacing. The combination of GA and MST constitutes a useful tool to design acoustic barriers based on SCs with no need for sophisticated scatterers. The technological advantages of using rigid cylinders as scatterers are their high resistance for use outdoors, their constructive simplicity, and their low cost due to the reduction in volume of the crystal.

This work has been supported by the Comisión Interministerial de Ciencia y Tecnología of Spain (Contract No. MAT 2003-04993-03) and by Generalitat Valenciana (Spain) under Grant No. GV04B-371.

${ }^{1}$ F. Cervera, L. Sanchis, J. V. Sánchez-Pérez, R. Martinez-Sala, C. Rubio, C. López, D. Caballero, and J. Sánchez-Dehesa, Phys. Rev. Lett. 88, 023902 (2002).

${ }^{2}$ L. Sanchis, A. Hakansson, F. Cervera, and J. Sánchez-Dehesa, Phys. Rev. B 67, 035422 (2003).

${ }^{3}$ R. Martínez-Sala, J. Sancho, J. V. Sánchez, V. Gómez, J. Llinares, and F. Meseguer, Nature (London) 387, 241 (1995).

${ }^{4}$ J. V. Sánchez-Pérez, D. Caballero, R. Martínez-Sala, C. Rubio, J. SánchezDehesa, F. Meseguer, J. Llinares, and F. Gálvez, Phys. Rev. Lett. 80, 5325 (1998).

${ }^{5}$ F. R. Montero de Espinosa, E. Jiménez, and M. Torres, Phys. Rev. Lett. 80, 1208 (1998)

${ }^{6}$ D. Caballero, J. Sánchez-Dehesa, R. Martínez-Sala, C. Rubio, J. V. Sánchez-Pérez, L. Sanchis, and F. Meseguer, Phys. Rev. B 64, 064303 (2001).

${ }^{7}$ Z. Liu, X. Zhang, Y. Mao, Y. Y. Zhu, Z. Yang, C. T. Chan, and P. Sheng, Science 289, 1734 (2000).

${ }^{8}$ M. Hirsekon, P. P. Delsanto, N. K. Batra, and P. Matic, Ultrasonics 42, 231 (2004).

${ }^{9}$ M. Hirsekorn, Appl. Phys. Lett. 84, 3364 (2004).

${ }^{10}$ O. Umnova, K. Attenborough, and C. M. Linton, J. Acoust. Soc. Am. 119, 278 (2006)

${ }^{11}$ X. Hu, C. T. Chan, and J. Zi, Phys. Rev. E 71, 055601 (2005).

${ }^{12} \mathrm{~V}$. Tournar, V. Pagneux, D. Lafarge, and L. Jaouen, Phys. Rev. E 70, 026609 (2004).

${ }^{13}$ J. H. Holland, Adaptation in Natural and Artificial Systems (The University of Michigan Press, Ann Arbor, MI, 1975).

${ }^{14}$ Z. Ye and Y. Chen, Phys. Rev. E 64, 036616 (2001).

${ }^{15}$ L. Sanchis, F. Cervera, J. Sánchez-Dehesa, J. V. Sánchez-Pérez, C. Rubio, and R. M. Martinez-Sala, J. Acoust. Soc. Am. 109, 2598 (2001).

${ }^{16}$ A. Hakansson, J. Sánchez-Dehesa, and L. Sanchis, Phys. Rev. B 70, 214302 (2004).

${ }^{17}$ L. Sanchis, A. Hakansson, D. López-Zanón, J. Bravo-Abad, and J. Sánchez-Dehesa, Appl. Phys. Lett. 84, 4460 (2004).

${ }^{18}$ A. Hakansson, F. Cervera, and J. Sánchez-Dehesa, Appl. Phys. Lett. 86, 054102 (2005). 